\title{
Hadronic Nuclear Energy: An Approach Towards Green Energy
}

\author{
Indrani B. Das Sarma \\ Department of Applied Chemistry, Jhulelal Institute of Technology, Lonara, Nagpur, India
}

Email address:

dassarmaindrani@rediffmail.com

\section{To cite this article:}

Indrani B. Das Sarma. Hadronic Nuclear Energy: An Approach Towards Green Energy. American Journal of Modern Physics. Special Issue: Issue II: Foundations of Hadronic Mechanics. Vol. 5, No. 2-1, 2016, pp. 119-130. doi: 10.11648/j.ajmp.2016050201.16

Received: August 11, 2015; Accepted: August 12, 2015; Published: June 1, 2016

\begin{abstract}
Nuclear energy is undoubtedly the largest energy source capable of meeting the total energy requirements to a large extent in long terms. However the conventional nuclear energy involves production of high level radioactive wastes which possesses threat, both to the environment and mankind. The modern day demand of clean, cheap and abundant energy gets fulfilled by the novel fuels that have been developed through hadronic mechanics / chemistry. In the present paper, a review of Prof. R. M. Santilli's Hadronic nuclear energy by intermediate controlled nuclear synthesis and particle type like stimulated neutron decay and double beta decay has been presented.
\end{abstract}

Keywords: Intermediate Controlled Nuclear Synthesis, Stimulated Neutron Decay

\section{Introduction}

The ever increasing demand for good quality of livelihood has ultimately culminated in increasing global energy demands. The demand can be met conventionally by either molecular combustion or nuclear fission. The former is achieved by combustion of fossil fuel or hydrogen which produces large amount of green house gases as well as depletes breathable oxygen from the environment. The latter does not generate green house gases or depletes breathable oxygen but creates large amount of radioactive wastes. Moreover, the shielding from the high energy ionizing and non-ionizing radiations is cumbersome and expensive. The handling of the highly radioactive wastes posses environmental as well as security threat. Thus, handling of these wastes requires great deal of safety requirements. There are several ways that are used to curb the either menace such as using better furnace design, improvising fuels and additives for molecular combustion or improvising fuel geometry and reactor design for efficient nuclear fission. In either case the perilous waste products are not completely eliminated. Although there are energy sources that have zero emissions like the energy harnessed from renewable sources like solar, wind, tidal, geo-thermal, wave, ocean-thermal and so on but are mainly time and location dependent. Hence cannot be universally employed for harnessing energy or power generation.
On the other hand, the nucleus of an atom has always been considered to be the source of unlimited energy since its discovery in 1911 by Ernest Rutherford [1]. The basic nuclear processes are of two types viz., fission and fusion. Both these processes generate large amount of energy which can be conveniently harnessed for useful work. The fission reaction is exoergic and criticality can be attained easily but fusion is endoergic and achieving criticality is comparatively difficult. Hence fission has been extensively explored for destructive as well as constructive work.

The unlimited source of the atomic nucleus due to fission process was initially exclusively exploited for destructive purpose. However, post World War II the focus shifted more towards constructive work. Attention was turned to the peaceful and directly beneficial application of nuclear energy. In the course of developing nuclear weapons the Soviet Union and rest of the Western world had discovered range of new technologies. Scientists also realized that the tremendous heat produced in the process could be tapped either for direct use or for generating electricity. It was also clear that this new form of energy had tremendous potential for the development of compact long-lasting power sources which could have various applications.

The world's first artificial nuclear reactor was Chicago Pile-1. It was a research reactor. Its construction was a part of the Manhattan Project. It was carried out by the Metallurgical 
Laboratory, University of Chicago under the supervision of Enrico Fermi, alongwith Leó Szilárd (discoverer of the chain reaction), Martin Whittaker, Walter Zinn and George Weil [2, 3]. The first man-made self-sustaining nuclear chain reaction was initiated in Chicago Pile-1 on December 2, 1942. The apparatus was described as a crude pile of black bricks and wooden timbers by Fermi. The pile contained large amount of graphite $(771,000 \mathrm{lbs})$ and uranium $(80,590 \mathrm{lbs}$ of uranium oxide and $12,400 \mathrm{lbs}$ of uranium metal). The pile was in the form of flattened ellipsoid measuring 25 feet wide and 20 feet high. The neutron producing uranium pellets were separated from one another by graphite blocks in the pile. Some of the free neutrons produced by the natural decay of uranium were absorbed by other uranium atoms, causing nuclear fission of those atoms and the release of additional free neutrons. The graphite between the uranium pellets was neutron moderator that thermalized neutrons, increasing fission cross-section. The control rods were of cadmium, indium (for preventing uncontrolled chain reaction) and silver (measuring the flux). Unlike the modern reactors, it lacked radiation shield or cooling system.

The first nuclear reactor to produce electricity by fission was the Experimental Breeder Reactor-I or Chicago Pile-4 designed and operated by Argonne National Laboratory, Idaho, USA under the supervision of Walter Zinn. This LMFBR went critical in December 1951. It produced $0.8 \mathrm{~kW}$ in a test run on December 20, 1951[4] and $100 \mathrm{~kW}$ of electrical power the following day, [5] having a design output of $200 \mathrm{~kW}$ of electrical power.

With advancement of technologies, the modern fission reactors have high energy output but have disadvantages such as enrichment of fuel and / or moderator; disposal of high energy radioactive waste and cumbersome shielding from high energy ionizing radiations. Thus, the energy harnessed is not completely green. On the other hand fusion process does not generate large amount of nuclear waste and if nuclei combine at threshold energy then the chances of crossing the fission barrier and emission of ionizing radiation are reduced considerably.

\section{Nuclear Fusion}

The nuclear fusion has always been considered the holy grail of unlimited clean energy. The reason for this is probably the thermonuclear reactions taking place in the sun and other stars [6]. In this case, nuclear fusion is achieved by using extremely high temperatures. The average kinetic energy of the combining nuclei increases proportionately with temperature. The temperature is determined by Lawson criteria [7] as given by expression 1 .

$$
n \tau_{E} \geq L \equiv \frac{12}{E_{c h}} \frac{k_{B} T}{\langle\sigma v\rangle}
$$

where, $n, \tau_{E}, E_{c h}$ and $k_{B}$ are particle density, confinement time, energy of charged fusion product and Boltzmann constant respectively.

The quantity $T /\langle\sigma v\rangle$ is a function of temperature with an absolute minimum. Replacing the function with its minimum value provides an absolute lower limit for the product $n \tau_{E}$. This is the Lawson criterion. At the temperature predicted by the Lawson criterion the energy of the colliding particles confined within the plasma are high enough to overcome the Coulomb barrier and chances of fusion increases. The colliding nuclei are confined within the plasma by gravitational or magnetic or inertial confinement. The controlled thermonuclear fusion reactions take place in an environment allowing some of the resulting energy to be harnessed for constructive purposes. Since this reaction takes place at very high temperature, so is popularly known as Hot Fusion. The major drawback is that it is not self sustaining and compound nucleus undergoes fission leading to formation of radioactive wastes. This is because the atomic electron clouds are completely stripped off. Kinetic energies of the colliding nuclei are increased to overcome the coulombic barrier and the energy attained by the compound nucleus is generally higher than the fission barrier which results in fission reaction or nuclear decay as prominent exit channels.

Fleischmann, Pons and Hawkins [8] in the year 1989 reported their historic but the most debatable findings. They observed unusual excess heat in the electrolysis of heavy water using deuterium loaded palladium electrodes. This they presumed to be due nuclear fusion reaction. Since the reaction taking place is at low temperature, they termed it as cold fusion on similar terms as hot fusion. Cold fusion or low energy nuclear fusion is known to occur under certain conditions in metal hydrides. It produces excess heat and nuclear ash such as helium, charged particles and sometimes very low level of neutrons. In certain cases the host metal has been found to be transmuted into other elements. The cold fusion reaction has been reported with palladium, titanium, nickel, some superconducting ceramics and so on. It has been observed due to varied triggers like ultrasonic waves, laser beam, electrical current. The major explanation for this phenomenon is reported to be the induction of electrostatic pressure to the reacting nuclei within the lattice of the metal. This environment is difficult to achieve and hence the phenomenon is non-reproducible. This could be due to insufficient energy required to expose the atomic nuclei from within the covering atomic electron cloud.

\subsection{Nuclear Processes and Quantum Mechanics}

Quantum mechanics is based on Galilei and Poincaré symmetries [9]. They are applicable only for Keplerian systems, where the various particles orbit around a centrally located nucleus, such as planets around central star / sun or electrons around nucleus. However, quantum mechanics is not applicable in understanding interaction between those particles which lack such symmetries like interaction between two electrons in a sigma bond or lateral overlap as in $\pi$-bonds. The Hamiltonian nature of quantum mechanics restricts the understanding of nuclear forces. Hence, to represent the nuclear force with a potential up to 35 different potentials have been added without achieving the required exact representation. The linear, local and Hamiltonian character of quantum mechanics is effective for the classification of 
hadrons under their point-like approximation, but is inadequate for structure related problems due to expected nonlinear, nonlocal and non-Hamiltonian effects occurring within the hyper dense media inside hadrons.

Thus, Prof. Santilli [10] states: According to the standard model, at the time of the neutron synthesis from protons and electrons inside a star, the permanently stable protons and electrons simply disappear from the universe to be replaced by conjectural quarks, and then the proton and the electron simply reappear at the time of the neutron decay. These beliefs are simply repugnant to me because excessively irrational, thus showing the conduction of particle physics via academic authority, rather than scientific veritas.

The quantum theory fails to explain the following even for the simplest nucleus of deuterium $[9,10]$ -

1. The spin 1 of deuterium since quantum axioms require that the single stable bound state of two particles with spin $1 / 2$, (proton and neutron) must be the singlet state with spin zero.

2. To represent the magnetic moment of deuterium.

3. The stability of unstable neutron when coupled to proton in a nucleus (e.g. deuterium).

$\mathrm{T}_{1 / 2}$ of neutron $\cong 15$ minutes.

4. Quantum Mechanics is inapplicable for explaining the synthesis of neutron from a proton and an electron as occurring in stars because; in this case the Schrödinger equation becomes inconsistent.

It is unsuitable for all processes that are irreversible over time, like nuclear fusions, because quantum mechanics is reversible over time, thus admitting the time reversal event which violates energy conservation, causality and other basic laws.

\subsection{Hadronic Mechanics}

Quantum mechanics was conceived for the study of interactions among particles at large mutual distances which is represented with differential equations defined over a finite set of isolated points. It does not have the scope for the study of the additional nonlocal-integral interactions due to mutual wave overlapping. These interactions are defined over an entire volume and cannot be effectively approximated by their abstraction into finite number of isolated points. Thus, the same cannot be derived from a Hamiltonian or their derivatives [9].

Thus, Prof. Santilli has founded more fundamental theory of the universe, named after the composite nuclear particle hadron as Hadronic Mechanics. Hadronic mechanics was formulated as an extension of quantum mechanics, encompassing its insufficiencies for the study of the additional nonlocal-integral interactions due to mutual wave overlapping. Thus the range of hadronic, quantum and classical mechanics can be depicted as in Figure 1.

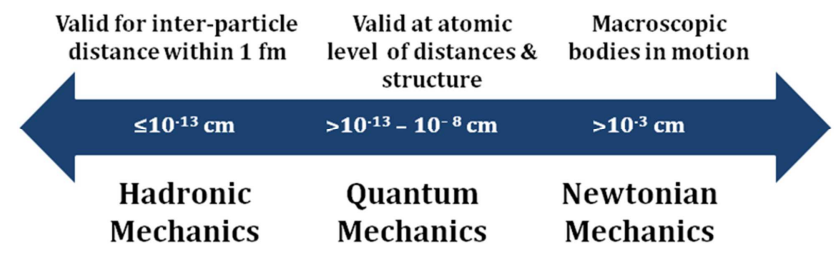

Figure 1. Various range of validity for Hadronic, Quantum and Newtonian Mechanics.

The emergence of strongly attractive force for deeply overlapping particles is one of the fundamental contributions of hadronic mechanics. There are varied instances where hadronic mechanics could satisfactorily explain the interactions such as quantitative treatment of neutron synthesis from protons and electrons (as occurring in stars), nuclear fusion, explanation of nuclear structure, strong nuclear binding energy, strong interaction between two electrons in a sigma bond, formation of magnecular bonds, formation of cooper pair in superconductors, and so on. Thus, hadronic mechanics could provide a quantitative treatment for the possible utilization of inextinguishable energy contained inside the neutron and formation of light nuclei. In other words, the study of new clean energies and fuels that cannot even be conceived with the $20^{\text {th }}$ century doctrines and other basic advances can be done with the new sciences. So, hadronic mechanics is rightly called as new sciences for new era [10].

The modern day demand of clean, cheap and abundant energy source can be fulfilled by changing the approach from quantum mechanics to hadronic mechanics to hadronic chemistry. In view of this, Prof. Ruggero Maria Santilli proposed various types of new non-nuclear as well as nuclear fuels. Non-nuclear fuels are basically magnecules that show magnecular combustion similar to conventional molecular combustion albeit cleaner, greener and with higher calorific values probably due to stored magnetostatic energy within the magnecules $[12,13]$. The hadronic fuels are summarized in Figure 2.

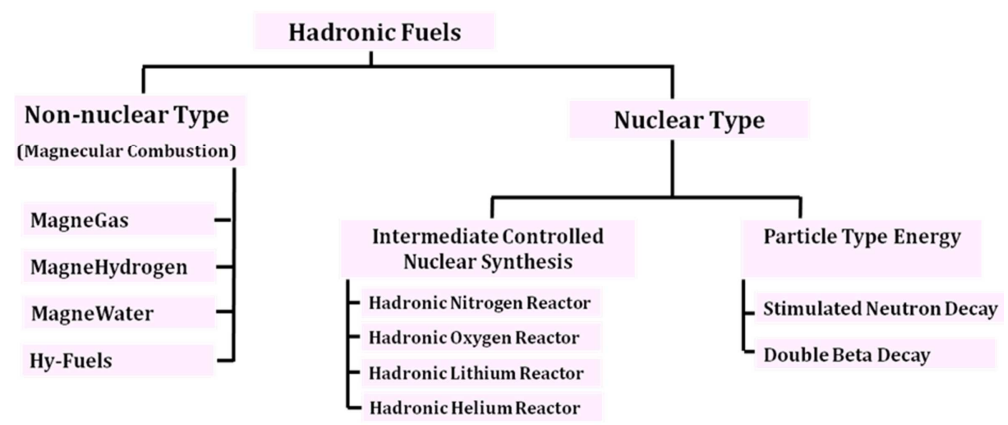

Figure 2. The classification of various hadronic fuels. 


\subsection{Nuclear Type Hadronic Fuels}

The nuclear fuels proposed by Prof. Santilli under hadronic mechanics are controlled nuclear reactions (fusion as well as fission) without ionizing radiations and radioactive waste. The nuclear fission could be adequately explained by quantum mechanics by considering the fragments as point mass. However, the same theory fails to explain nuclear fusion because considering the reacting nuclei as point mass is impractical [10]. Nucleus is a hyper dense medium containing protons and neutrons. Since neutrons are made up of protons and electron, hence Prof. Santilli projects nucleus of an element as collection of mutated protons and electrons. The basic assumptions $[11,12]$ proposed by Prof. Santilli are-

1. Nuclear force: Nuclear force was initially considered to be derived completely from a potential. So it was represented with a Hamiltonian. However, Prof. Santilli assumed that nuclear force is partly of action-at-a-distance, potential type that can be represented with a Hamiltonian and partly is of contact, non-potential type that cannot be represented with a Hamiltonian. This assumption implies that the time evolution of nuclear structure and processes is essentially of non-unitary type. So the use of hadronic mechanics is mandatory as it is the only known axiomatically consistent and time invariant non-unitary formulations of nuclear structures and their processes.

2. Stable nuclei: Nuclei have no nuclei of their own and are composed of particles in contact with each other having mutual penetration of about $10^{-3}$ of their charge distributions shown in Figure 3. So, the nuclear force is expected to be partially of potential and partially of non-potential type, with ensuing non-unitary character of the theory, and related applicability of hadronic mechanics.

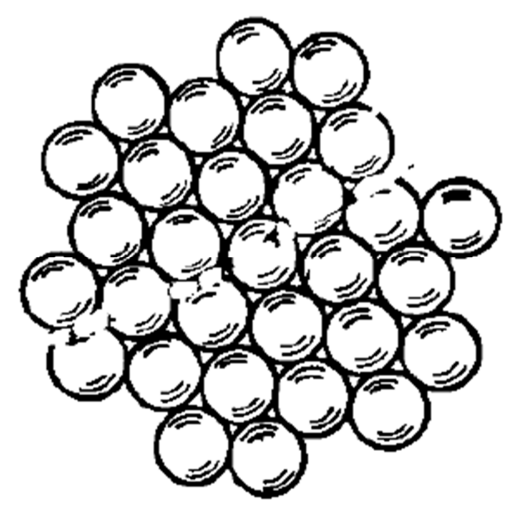

Figure 3. Schematic representation used by Prof. Santilli to illustrate that nuclei have no nuclei of their own and are composed of particles in contact with each other.

Let $\mathrm{A}$ be the time evolution of a Hermitean operator in the infinitesimal and finite forms derived from Heisenberg-Santilli Lie-isotopic equations proposed in 1978 by Prof. Santilli for stable, reversible, interior dynamical problems.

$$
\begin{aligned}
& i \frac{d A}{d t}=[A, H]=A T H-H T A \\
& A(t)=\exp (H T t i) A(0) \exp (-i t T H)
\end{aligned}
$$

where the Hermitean Hamiltonian

$H=\frac{p^{2}}{2 \mathrm{~m}}+V(r)$ represents all possible nuclear forces truly derived from potential $\mathrm{V}(\mathrm{r})$;

Isotopic element $T$ represents all contact non-potential interactions allowing the nuclear structure with all constituents in actual contact with each other, and the simplest possible realizations of type

$$
T=\exp \left(-F(r) \int \psi^{\dagger}(r) \psi(r) d^{3} r\right)>0
$$

which recovers quantum mechanics when there is no appreciable overlapping of the wavefunctions $1 \psi$ of nuclear constituents; and the inverse of isotopic element

$$
I=\frac{1}{T}>0
$$

represents the basic, right and left unit of the theory at all levels, non-zero values of $T$ depicts non-Hamiltonian interactions (presence of contact).

The stability of the nucleus (reversibility over time) is represented by the identity of the basic iso-unit to the right and to the left, namely, for motions forward and backward in time.

3. Unstable nuclei and nuclear fusion: According to the Heisenberg-Santilli Lie-admissible equations for the time evolution of Hermitean operator $A$ in their infinitesimal and finite forms

$$
i \frac{d A}{d t}=(A, H)=A R H-H S A
$$

where $H$ is Hermitean representing the non-conserved total energy

genotopic elements $R$ and $S$ represents the non-potential interactions.

Irreversibility is depicted by the different values of the genounit for forward (f) and backward (b) motions in time

$$
I=\frac{1}{R} \neq I=\frac{1}{S}
$$

Here, the Lie-admissible branch of hadronic mechanics is ideally suited to represent the decay of unstable nuclei as well as nuclear fusions, since both are irreversible over time.

4. Neutron synthesis: Rutherford's conjecture on neutron as a compressed hydrogen atom was experimentally verified later by Don Borghi's experiment. It is also well-known that synthesis of neutron from the compressed hydrogen gas is precursor to synthesis of all natural elements in a star. So, the synthesis of the neutron is the most fundamental nuclear synthesis. As shown in Figure 4 (a) the original drawing used by Prof. Santilli to illustrate the physical difference between the hydrogen atom and neutron synthesis from proton and 
electron. The figure 4 (b) depicts the additional non-linear, non-local and non-potential interactions due to deep wave overlapping of proton and electron in neutron which is otherwise absent in hydrogen atom. This non-Hamiltonian character requires a non-unitary extension of quantum mechanics.
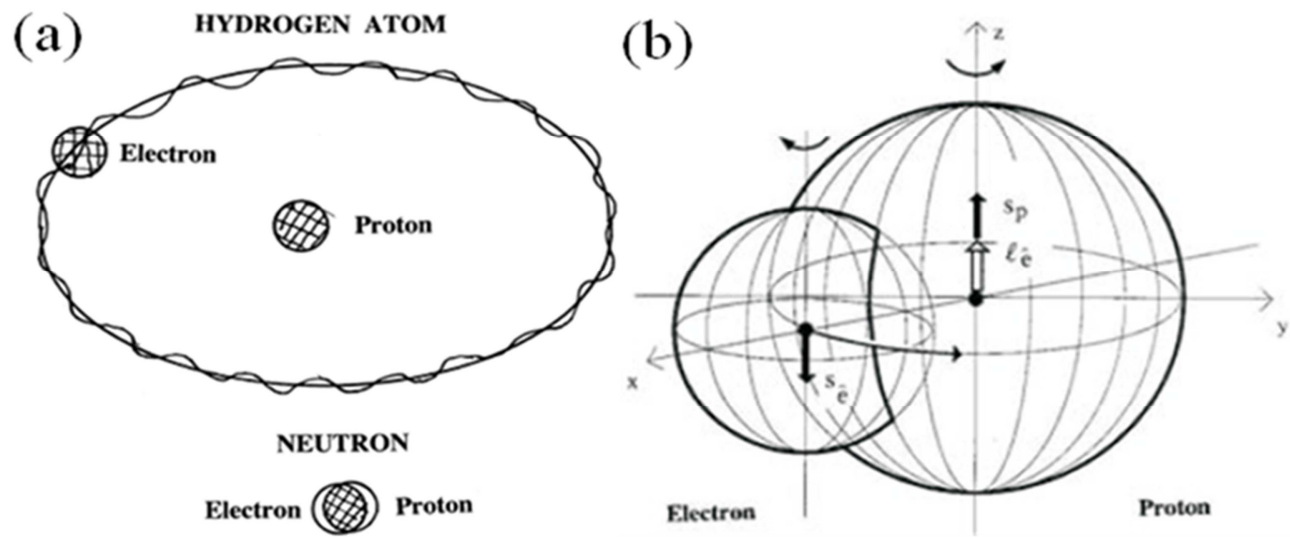

Figure 4. (a) Original drawing used by Prof. Santilli to illustrate the physical difference between the hydrogen atom and neutron. (b) additional non-linear, non-local and non-potential interactions due to deep wave overlapping of proton and electron in neutron.

Consequently, Prof. Santilli quantified neutron synthesis using hadronic mechanics as

$$
p^{+}+a+e^{-} \rightarrow n
$$

where ' $a$ ' is Santilli's etherino which is a conventional Hilbert space the transfer of $0.782 \mathrm{MeV}$ and spin $1 / 2$ missing in the neutron synthesis from the environment to the neutron structure.

The etherino disappears at the covering level of hadronic mechanics and the neutron synthesis on a iso-Hilbert space over an iso-fields. Finally, the missing $0.782 \mathrm{MeV}$ energy required for the synthesis of the neutron is provided by the environment. For instance, a star would not start producing light due to huge amount of energy needed for the synthesis of large number of neutrons. Thus, it was concluded that for continuous creation of neutron in the universe the missing energy is provided by the ether as a universal substratum.

5. Nuclear Structure: Prof. Santilli assumes the unitary classification of baryons as valid, but introduces new structure models of each member of the baryonic family with physical constituents that can be produced free, thus being detected in the spacetime. Resolution of historical objections is merely achieved by assuming that, when in interior conditions (only), barionic constituents obey hadronic mechanics and symmetries with subsequent mutations (denoted by hat) of their intrinsic characteristics. Proton is assumed to be an elementary stable particle without known structure and neutron to be an unstable narticle comprising of a proton $\hat{p}^{+}$and an electron $\hat{e}^{-}$in mutated conditions due to their total mutual immersion and resulting synthesis

$$
n=\left(\hat{p}^{+}, \hat{e}^{-}\right)_{h m}
$$

As a result, it is assumed that nucleus is a collection of protons and neutrons in first approximation, while being at a deeper level it is a collection of mutated protons and electrons.

\subsubsection{Controlled Nuclear Synthesis (CNS)}

The hot fusion or cold fusion reactions are difficult to achieve. The high temperature required for hot fusion and random occurrence of cold fusion limits their use for economic energy output. One of the major successes of hadronic mechanics and iso sciences is their ability to obtain industrial realization of fusion reactions without any ionizing radiations. These reactions are controlled as well as have intermediate energy requirements than hot or cold fusions hence are called as controlled nuclear synthesis (CNS) or intermediate controlled nuclear synthesis (ICNS). Controlled Nuclear Synthesis (CNS) are given by systematic energy releasing nuclear fusions whose rate of synthesis (or of energy output) is controllable via one or more mechanisms capable of performing the engineering optimization of the applicable laws $[11,12]$.

There are various physical laws which are to be obeyed by all nuclear fusions to occur in a systematic way rather than in a random way. The CNS is governed by Santilli's laws for controlled nuclear synthesis $[11,12]$ :

1. The orbitals of peripheral atomic electrons are controlled such that nuclei are systematically exposed. Nuclei are shielded by the electron cloud. It is obvious that nuclear synthesis between two atoms is impossible at low energies because the electron cloud restricts approachability of the interacting nuclei. This law explains the inability of the cold fusions to achieve energy output of industrial significance because in this case the energy necessary for systematic exposure of nucleus from electron cloud is low. This law also emphasises the need for the proposed intermediate synthesis in which the first energy requirement is precisely the control of atomic clouds.

2. CNS occurs when nuclei spins are either in singlet planar coupling or triplet axial coupling. This law shows the structural difference between quantum and 
hadronic mechanics. The constituents of a bound state of two quantum particles must be point-like to avoid structural inconsistencies such as local-differential topology. As a result, as per quantum mechanics singlet and triplet couplings are equally possible. However, when the actual extended character of the constituents is taken into account, it is clear that triplet planar couplings of extended particles at short distances are strongly repulsive, while singlet planar couplings are strongly attractive. Planar means that the two nuclei have a common median plane and axial means a common axial symmetry as shown in figure 5 . This law was the basis to build hadronic mechanics via gear model. In fact, the coupling of gears in triplet (parallel spins) causes extreme repulsion, while the only possible coupling of gears is in singlet (antiparallel spins). The emergence of strongly attractive force for the singlet planar or triplet axial couplings is one of the fundamental contributions of hadronic mechanics to fusion processes since such a force is totally absent for quantum mechanics, while it appears naturally in all spinning and deeply overlapping particles.
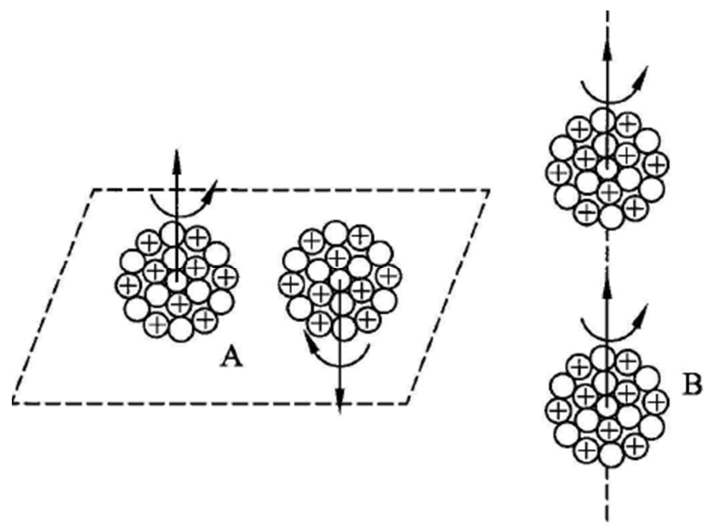

Figure 5. Schematic representations of the only two stable couplings permitted by hadronic mechanics for nuclear synthesis; the singlet planar coupling (A) and the triplet axial coupling (B). All other spin configurations have been proved to produce strongly repulsive forces under which no CNS is possible.

3. The most probable CNS are those occurring at threshold energies and without the release of massive particles or ionizing radiations. In other words, CNS occurring at threshold energies are green in nature as they do not emit ionizing radiations or ejectiles. The threshold energy mostly hinders fusion reaction. If the energy is lower than the threshold energy then industrially meaningful nuclear syntheses is not possible as in case for cold fusions, although random synthesis may occur due to tunnelling effect. On the other hand, if the energy of the interacting nuclei is higher than the threshold energy then the excess energy is reflected as excitation energy of the resulting compound nucleus. Thus, excitation energy of the compound nucleus is directly proportional to the energy of the interacting nuclei. The excitation energy is dissipated by emission of gamma photon or particles or fission of the resulting compound nucleus as shown in figure 6.

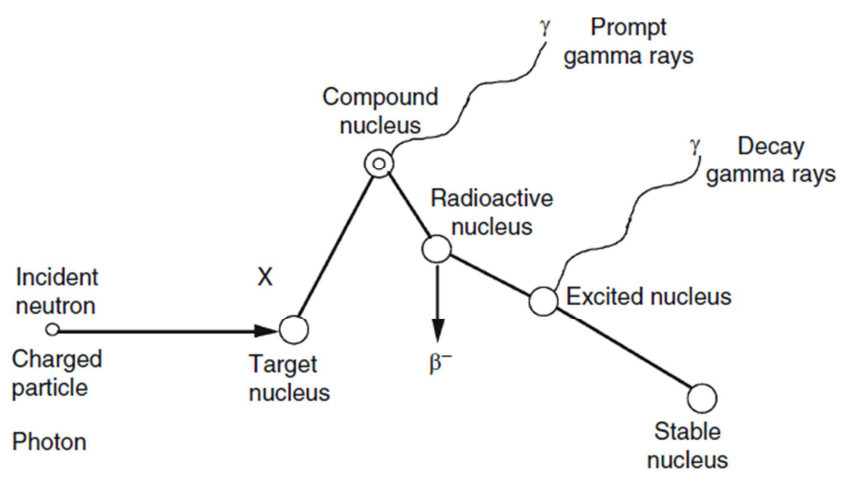

Figure 6. Formation of compound nucleus having high excitation energy.

The calculations based on hadronic mechanics indicate that the probability of a nuclear synthesis with the release of neutrons is much smaller than that of synthesis without the emission of massive particles. This law has been verified by ICNS data and also it appears to be verified by nuclear syntheses spontaneously occurring in nature. However, this does not mean that CNS with secondary emission is impossible. This only suggests that the nuclear synthesis could be green which was earlier unimaginable.

4. CNS requires trigger, an external mechanism that forces exposed nuclei to come in fm range (hadronic horizon). All nuclei are positively charged, thus repel each other at distances bigger than one Fermi. Nuclear synthesis is impossible without overcoming the coulombic repulsion that brings nuclei inside the hadronic horizon. Inside the hadronic horizon, the preceding laws are verified (particularly second law on spin couplings). The synthesis is inevitable due to the activation of the strongly attractive hadronic forces (typical non-potential interaction) that overcome the repulsive Coulomb force.

Considering the Fleishmann-Pons electrolytic cell in purview of Santilli's Law of CNS, it is clear that this cell does verify the conservation of the energy, angular momentum and has a trigger characterized by the electrostatic pressure compressing deuterium inside the palladium. However, Fleishmann-Pons electrolytic cell does not verify first law (control of atomic clouds to expose nuclei) and second law (control of spin couplings). Here the nuclear spin couplings occur at random; there is lack of identified mechanism for systematic exposure of the interacting nuclei and optimization of the verified laws. Consequently, nuclear syntheses occur at random, preventing economic values of the energy output. Thus, it is evident that for nuclear synthesis of economic value to occur all the above laws should be verified.

\subsubsection{Magnecules: A Precursor for Nuclear Synthesis}

Magnecules proposed by Prof. R. M. Santilli is a novel chemical species that have at least one magnecular bond between two atoms or radicals or molecules. The atoms are 
held together by magnetic fields originating due to toroidal polarization of the atomic electron orbits [13, 14]. The rotation of the electrons within the toroid creates the fifth field force, the magnetic field, which cannot originate for the same atom if the conventional spherical electron distribution in orbitals is a physical reality. When two such polarized atoms are sufficiently close to each other and in north-south north-south alignment, the resulting total force between the two atoms is attractive as shown in Fig.7.

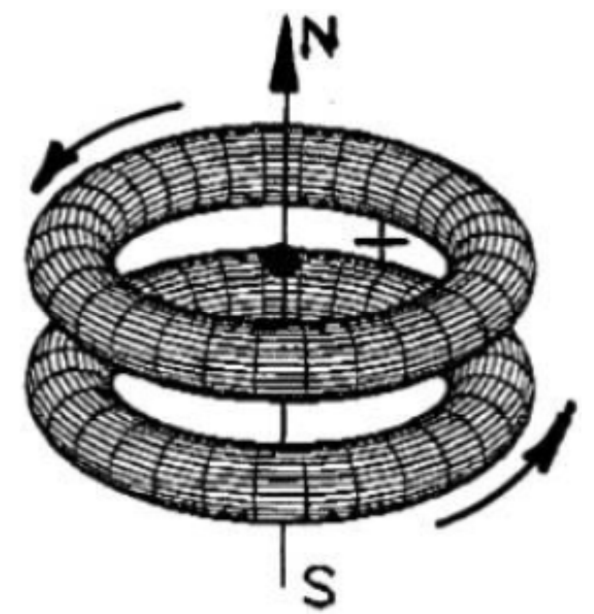

Figure 7. Conceptual depiction of an elementary magnecule comprising two identical or different atoms whose bond is entirely of magnecular character, namely, originating from opposing polarities North-South-North-South of the toroidal distributions of orbitals, as well as the polarization of nuclear and electron magnetic moments.

Thus, the simple principle of synthesizing magnecules is similar to the magnetization of a ferromagnet where the orbits of unbounded electrons are polarized. The added beauty of magnecules is that the nucleus is systematically exposed and the two nuclei can approach each other without appreciable columbic barrier. The internuclear distance is governed by the bond energy of the magnecular bond. The nuclei of the interacting atoms approach more closely than in case of conventional molecular bond allowing the required singlet planar or triplet axial coupling for nuclear synthesis. Thus, when a trigger brings the two nuclei within 1 fm range (hadronic horizon) the fusion becomes inevitable and a new nucleus is formed. Since the parent nuclei are not having high energy the resulting daughter nuclei also does not possess high excitation energy, consequently there is no nuclear emission. Thus the process is green.

Thus, the ICNS proposed by Santilli are of the generic type $[11,12]$

$$
\begin{aligned}
& \mathrm{N}_{1}\left(\mathrm{~A}_{1}, \mathrm{Z}_{1}, \mathrm{~J}_{1}^{\mathrm{P}_{1}}, \mathrm{u}_{1}\right)+\mathrm{N}_{2}\left(\mathrm{~A}_{2}, \mathrm{Z}_{2}, \mathrm{~J}_{2}^{\mathrm{P}_{2}}, \mathrm{u}_{2}\right)+\mathrm{TR} \\
& \rightarrow \mathrm{N}_{3}\left(\mathrm{~A}_{3}, \mathrm{Z}_{3}, \mathrm{~J}_{3}^{\mathrm{P}_{3}}, \mathrm{u}_{3}\right)+\text { Heat } \\
& \text { where } \mathrm{A}_{1}+\mathrm{A}_{2}=\mathrm{A}_{3}, \quad \mathrm{Z}_{1}+\mathrm{Z}_{2}=\mathrm{Z}_{3}, \\
& \mathrm{~J}_{1}+\mathrm{J}_{2}=\mathrm{J}_{3}, \quad \mathrm{P}_{1}+\mathrm{P}_{2}=\mathrm{P}_{3}
\end{aligned}
$$

$A$ is the atomic number

$\mathrm{Z}$ is the nuclear charge

$\mathrm{J}^{\mathrm{P}}$ is the nuclear angular momentum with parity $\mathrm{u}$ is the nuclear energy in amu units

TR is trigger mechanism (high voltage DC arc in hadronic reactor) and mass defect is observed in form of heat

Nuclear synthesis via green mechanism is known to occur silently in nature [11]. This can be verified from the chemical analyses of about one hundred million years old amber sample. The trapped air bubbles showed 40\% nitrogen, whereas the current percentage of nitrogen in atmosphere is approximately $80 \%$. Other chemical analyses verify the above analysis that the increase of nitrogen in our atmosphere has been gradual.

According to Prof. Santilli, these data indicate the natural synthesis of nitrogen from lighter elements. The most probable mode of nitrogen synthesis in nature seems to be initiated by lightning as quantitative explanation of thunder is impossible by conventional chemical reactions, thus requiring nuclear syntheses. Numerical explanation of thunder requires energy equivalent to hundreds of tons of explosives that simply cannot be explained via conventional processes due to the very small cylindrical volume of air affected by lightning and its extremely short duration of the order of nanoseconds. However, the nitrogen syntheses by lightning provide numerical explanation of thunder as well as the gradual increase of nitrogen in the atmosphere. Among all possible syntheses, the most probable one results in being the synthesis of nitrogen from carbon and deuterium.

According to Prof. Santilli, these data indicate the natural synthesis of nitrogen from lighter elements. The most probable mode of nitrogen synthesis in nature seems to be initiated by lightning as quantitative explanation of thunder is impossible by conventional chemical reactions, thus requiring nuclear syntheses. Numerical explanation of thunder requires energy equivalent to hundreds of tons of explosives that simply cannot be explained via conventional processes due to the very small cylindrical volume of air affected by lightning and its extremely short duration of the order of nanoseconds. However, the nitrogen syntheses by lightning provide numerical explanation of thunder as well as the gradual increase of nitrogen in the atmosphere. Among all possible syntheses, the most probable one results in being the synthesis of nitrogen from carbon and deuterium.

$$
\begin{array}{r}
\mathrm{C}\left(12,6,0^{+}, 12.0000\right)+\mathrm{D}\left(2,1,1^{+}, 2.0141\right) \\
+\mathrm{TR} \rightarrow \mathrm{N}\left(14,7,1^{+}, 14.0030\right)+\Delta \mathrm{E} \\
\Delta \mathrm{E}=0.0111 \mathrm{amu}=10.339 \mathrm{MeV}
\end{array}
$$

However, the amount of deuterium present in the atmosphere is negligible to justify thunder quantitatively. Here, Prof. Santilli emphasizes the synthesis of neutrons by lightning from protons and electrons.

The neutron synthesis is expected to be a pre-requisite for the synthesis of deuterium in atmosphere which in turn synthesizes nitrogen which justifies energy of the thunder quantitatively. The same synthesis has been reproduced in laboratory quantitatively by Prof. Santilli using hadronic reactor. 


\subsection{Synthesis of Nitrogen from Carbon and Deuterium by ICNS}

The fusion reaction/nuclear synthesis taking place in hadronic reactor using deuterium as fuel and carbon electrode have shown to yield clean energy without formation of any radioactive species or ionizing radiations $[15,16]$. This synthesis is of industrial importance because it yields $10^{10}$ BTU of energy per hour which is equivalent to $10^{30} \mathrm{ICNS}$ per hour. The electric arc of the hadronic reactor polarizes carbon and hydrogen atoms by forming $\mathrm{C} \times \mathrm{H} \times \mathrm{H}$ magnecule, having triplet axial spin coupling. Under a suitable trigger (either high DC voltage or any other suitable means) the magnecule $\mathrm{C} \times \mathrm{H} \times \mathrm{H}$ yield a nucleus with $\mathrm{A}=14, \mathrm{Z}=8, \mathrm{~J}^{\mathrm{P}}=1^{+}$. However, this is impossible as $\mathrm{O}(14,8)$ has spin $\mathrm{J}=0$ and any other nucleus of the above mentioned type does not exist.

So, Prof. Santilli postulated that the nature synthesizes a neutron from proton, electron and etherino as

$$
\begin{gathered}
\mathrm{C} \times \mathrm{H} \times \mathrm{H} \rightarrow \mathrm{C}(12,6,0)+2 \times \mathrm{p}^{+}+\mathrm{e}^{-}+\mathrm{a} \\
\rightarrow \mathrm{C}(12,6,0)+\mathrm{H}(2,1,1) \rightarrow \mathrm{N}(14,7,1)
\end{gathered}
$$

\section{Other Examples of ICNS}

\section{(i). Synthesis of Silicon from Oxygen and Carbon}

$$
\begin{gathered}
\mathrm{O}\left(18,8,0^{+}, 17.9991\right)+\mathrm{C}\left(12,6,0^{+}, 12.0000\right) \\
+\mathrm{TR} \rightarrow \mathrm{Si}\left(30,14,0^{+}, 29.9737\right)+\Delta \mathrm{E} \\
\Delta \mathrm{E}=0.0254 \mathrm{u}
\end{gathered}
$$

This nucleosynthetic reaction verifies all conservation laws. The controlled fusion of oxygen and carbon into silica was done using $\mathrm{CO}_{2}$ (green house gas) as hadronic fuel for the production of clean energy [11]. The whitish powder formed on the edge of carbon electrodes of the hadronic reactor suggests synthesis of silica. Hadronic reactor was filled up with $\mathrm{CO}_{2}$ at pressure. The DC arc efficiently separates it into $\mathrm{O}_{2}$ and $\mathrm{C}$. $\mathrm{O}_{2}$ and $\mathrm{C}$ burns to produce $\mathrm{CO}$ which in the presence of oxygen and an arc, reproduces $\mathrm{CO}_{2}$. Thus recovering the energy used for the separation of $\mathrm{CO}_{2}$. However, along with the conventional combustion, the hadronic reactor produces a net positive energy output due to the fusion of oxygen and carbon into silica [17].
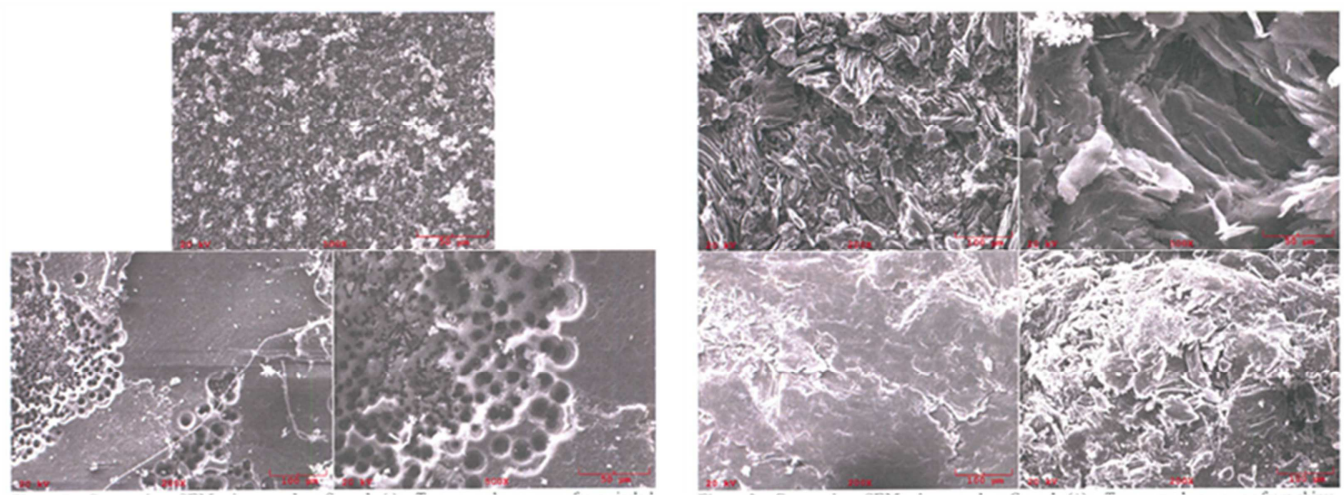

Figure 8. Semi-quantitative chemical compositions of the used electrodes were obtained using an Amray Scanning Electron Microscope equipped with an iXRF energy dispersive spectrometer (SEM-EDS) by Constellation Technology on $15^{\text {th }}$ October 2013. The elements detected were aluminium, silicon, calcium, chlorine and iron which were initially absent or if present were in trace amount.

\section{(ii). Synthesis of Oxygen from Carbon and Helium}

$$
\begin{array}{r}
\mathrm{C}\left(12,6,0^{+}, 12.0000\right)+\mathrm{He}\left(4,2,0^{+}, 4.0026\right) \\
+\mathrm{TR} \rightarrow \mathrm{O}\left(16,8,0^{+}, 15.9949\right)+\Delta \mathrm{E} \\
\Delta \mathrm{E}=0.0077 \mathrm{u}
\end{array}
$$

This nucleosynthetic reaction also verifies all conservation laws. Here, the interior of the reactor was cleaned, and various components replaced. A vacuum was pulled out of the inner chamber and the reactor was filled up with commercial grade helium at $100 \mathrm{psi}$. It was found that oxygen content decreased to a non-detectable amount but the $\mathrm{CO}$ increased from a non-detectable amount to $4.24 \%$.

The formation of $\mathrm{CO}$ depicts synthesis of oxygen at the tip of the DC arc hitting the carbon in the cathode surface. The resulting large local heat rapidly expels the synthesized oxygen from the DC arc, preventing any additional nuclear synthesis. However, high affinity of carbon and oxygen results in formation of carbon monoxide.

\section{(iii). Synthesis Silver from Palladium and Hydrogen}

$$
\begin{aligned}
& \operatorname{Pd}\left(106,46,0^{+}, 105.9034\right)+\mathrm{H}\left(1,1,1 / 2^{+}, 1.0078\right) \\
& +\mathrm{TR} \rightarrow \operatorname{Ag}\left(107,47,1 / 2^{+}, 106.90509\right)
\end{aligned}
$$

This nucleosynthetic reaction depicts the basic difference between pre-existing studies on cold fusion and the proposed ICNS. In this reaction palladium 106 is used as cathode and reactor is filled with hydrogen at a certain pressure.

If cold fusion occurs, then fusion reactions should take place inside the palladium cathode. However, the engineering implementation of the new CNS laws inside the palladium electrodes is virtually impossible, thus explaining the reason for the lack of its consideration in the industrial research. According to Prof. Santilli the nuclear fusions may occur in such conditions at random, thus preventing the controlled energy output necessary of industrial relevance.

Thus, albeit this reaction verifies conventional nuclear conservation laws but is not of industrial relevance owing to 
its random nature.

\subsection{Santilli Hadronic Reactors}

Hadronic reactors are the upgraded hadronic refineries originally designed by Prof. Santilli [11]. They use magnecular fuels for production of heat that can be used for power generation. The reactors house trigger mechanisms like high voltage DC arc or pressure impulse, etc as shown in Fig. 9 to facilitate controlled nuclear synthesis. Hadronic reactors can withstand higher pressure as compared to the hadronic refineries.

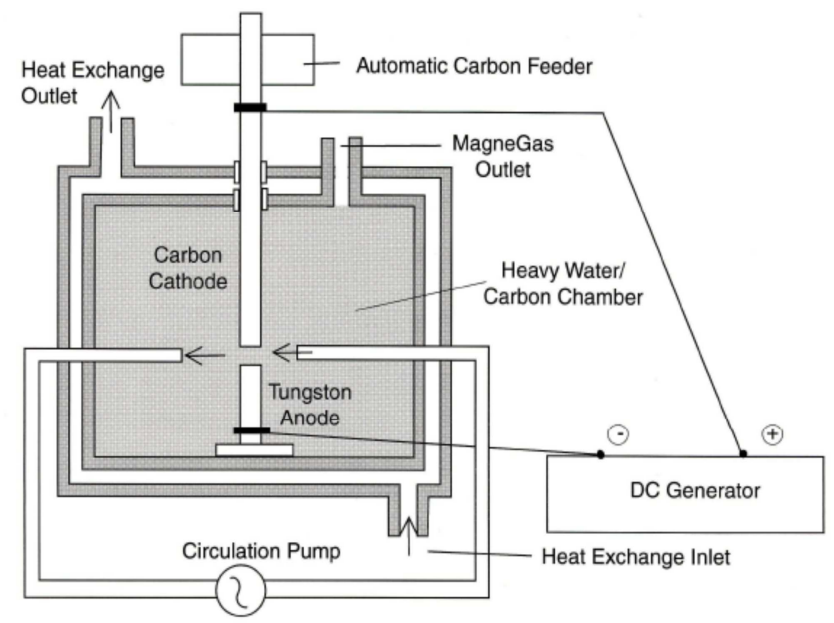

Figure 9. Schematic diagram of hadronic reactor based on an upgradation of the hadronic refineries showing emphasis on the production and use of a magnecular fuel in the latter, to the production and use of heat in the former.

These reactors are named based on the product or the fuel used. Hadronic nitrogen reactor is the most primitive type of hadronic reactor.

\subsubsection{Hadronic Nitrogen Reactor}

The reactor is filled with $\mathrm{D}_{2}$ gas at $3,000 \mathrm{psi}$ and is re-circulated through graphite electrodes. The trigger mechanism is by pulse DC arc of $100,000 \mathrm{~V}, 5 \mathrm{~mA}$ and other means. The heat is dissipated by the external heat exchanger. The heat is due to the nucleosynthetic reaction between deuterium and carbon occurring in reactor

$$
\begin{aligned}
& \mathrm{C}\left(12,6,0^{+}, 12.0000\right)+\mathrm{D}\left(2,1,1^{+}, 2.0141\right)+ \\
& \mathrm{TR} \rightarrow \mathrm{N}\left(14,7,1^{+}, 14.0030\right)
\end{aligned}
$$

\subsubsection{Hadronic Oxygen Reactor}

It is one of the simplest reactors as the reaction does not require spin polarizations for conservation of the angular momentum. So the reactor is similar to the one shown in Fig. 5 housing carbon electrodes. The vessel is filled up with a 50-50 mixture of oxygen 16 and helium at 3,000 psi, which is re-circulated through $50 \mathrm{~kW}$ electric arc to create magnecules of the type $\mathrm{O}$ He. The trigger is DC pulses of $100,000 \mathrm{~V}$ and $5 \mathrm{~mA}$, or by impulse pressures or other mechanisms. The heat produced is dissipated by the external heat exchangers.

Nucleosynthetic reaction occurring in the reactor

$$
\begin{gathered}
\mathrm{O}\left(16,8,0^{+}, 15.9949\right)+\mathrm{H}\left(2,1,1^{+}, 2.0141\right) \\
+\mathrm{TR} \rightarrow \mathrm{F}\left(18,9,1^{+}, 18.0009\right)+\Delta \mathrm{E} \\
\Delta \mathrm{E}=0.0081 \mathrm{u}=7.545 \mathrm{MeV}
\end{gathered}
$$

The instability of $\mathrm{F}\left(18,9,1^{+}, 18.0009\right)$ results in secondary process

$$
\begin{aligned}
& \mathrm{F}\left(18,9,1^{+}, 18.0009\right)+\mathrm{EC} \rightarrow \\
& \mathrm{O}\left(18,8,1^{+}, 17.9991\right)+1.656 \mathrm{MeV}
\end{aligned}
$$

Thus the total energy output per synthesis is equivalent to 9.201 MeV $1.30 \times 10^{-15}$ BTU.

If $10^{30}$ syntheses occur per hour then amount of green energy yielded would be substantial.

\subsubsection{First Hadronic Lithium Reactor}

First lithium reactor is the same as that of the oxygen reactor. The only difference is that the vessel is filled with $50-50$ mixture of hydrogen and helium gases at 3,000 psi. The mixture is also recirculated through a $50 \mathrm{~kW}$ electric arc that creates magnecules $\mathrm{H}$ He. The trigger is given by a high voltage pulse DC current or impulse pressure or other mechanism.

Nucleosynthetic reaction occurring in the reactor is

$$
\begin{aligned}
& \mathrm{H}\left(2,1,1^{+}, 2.0141\right)+\mathrm{He}\left(4,2,0^{+}, 4.0026\right)+ \\
& \mathrm{TR} \rightarrow \operatorname{Li}\left(6,3,1^{+}, 6.0151\right)+\Delta \mathrm{E} \\
& \Delta \mathrm{E}=0.0016 \mathrm{u}=2.50 \times 10^{-16} \mathrm{BTU}
\end{aligned}
$$

\subsubsection{Second Hadronic Lithium Reactor}

It is more complex than the first hadronic lithium reactor because of the need of lithium nuclei and a beam of protons with opposite polarization to avoid random reactions. The current technology allows a variety of engineering realizations of the needed polarization where a proton beam with down polarization enters a chamber of lithium with up polarization. Both polarizations are achieved via magnetic fields. The efficiency of the hadronic reactor depends on the geometry of the proton beams, the lithium chamber as well as required trigger.

Nucleosynthetic reaction occurring in the reactor

$$
\begin{array}{r}
\mathrm{Li}\left(7,3,3 / 2^{-}, 7.0160\right)+\mathrm{H}\left(1,1,1 / 2^{+}, 1.0078\right)+ \\
\mathrm{TR} \rightarrow 2 \times \mathrm{He}\left(4,2,0^{+}, 4.0026\right)+\Delta \mathrm{E} \\
\Delta \mathrm{E}=2.74 \times 10^{-15} \mathrm{BTU}
\end{array}
$$

Assuming efficiency of $10^{16}$ per minute one mole of lithium would produce energy equivalent to $1.7 \times 10^{6} \mathrm{~J}$ hour $^{-1}$.
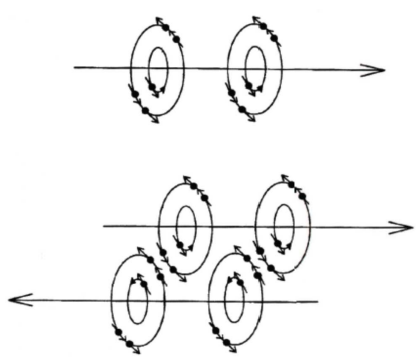

Figure 10. Schematic view of singlet (antiparallel) spin coupling required to synthesize helium from deuterium. 


\subsubsection{Hadronic Helium Reactor}

It is one of the most difficult as it requires the application of a trigger to two different beams of deuterium gas with opposite spin polarizations as depicted in Fig. 10. The reactor as shown in Fig. 11 is a metal vessel that houses two parallel but separate electric arcs with opposing polarities so as to produce opposite polarizations of the deuterium gas. The flow of the gas through said arcs from opposite directions creates the superposition of the beams in the area located between said arcs with spin couplings as shown in Fig. 11. The trigger seems to be the impulse pressure.

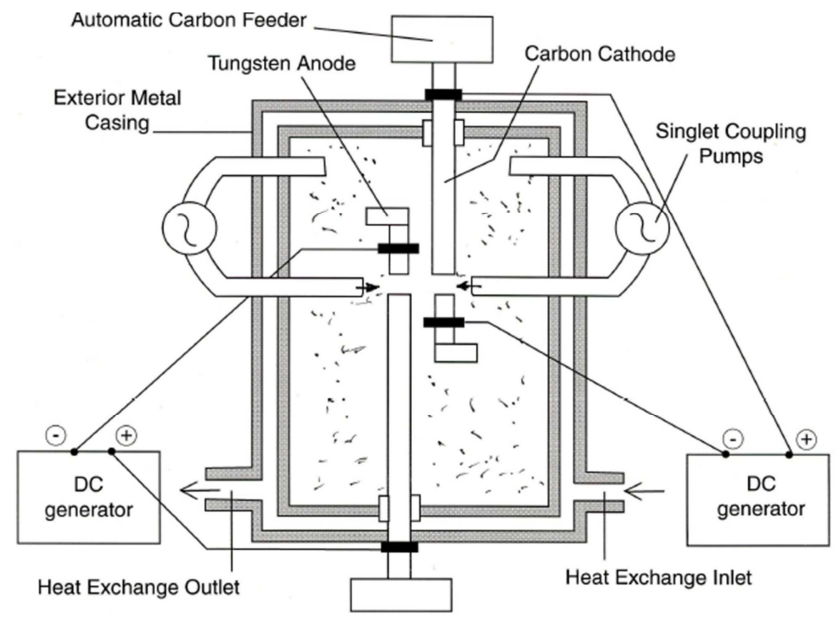

Figure 11. Schematic representation of hadronic reactor for nucleosynthesis of helium.

The nucleosynthetic reaction is-

$$
\begin{aligned}
& \mathrm{D}\left(2,1,1^{+} \uparrow, 2.0141\right)+\mathrm{D}\left(2,1,1^{+} \downarrow, 2.0141\right) \\
& +\mathrm{TR} \rightarrow \mathrm{He}\left(4,2,0^{+} \uparrow, 4.0026\right)+\Delta \mathrm{E}
\end{aligned}
$$

\subsection{Particle Type Hadronic Energy: Stimulated Decay of Energy}

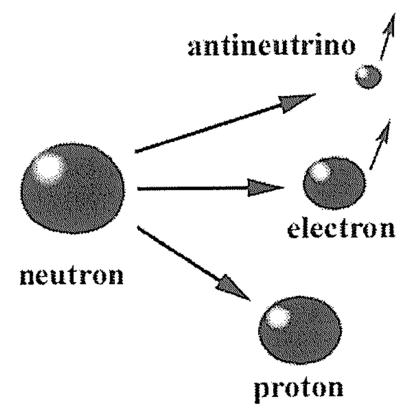

Figure 12. Schematic representation of stimulted neutron decay.

Hadronic nuclear energy can also be obtained by fission reactions or decay of stable nuclei. Theoretically any stable nuclei can be disintegrated into its nuclear constituents by photons having higher energy than the binding energy of the nuclei to be disintegrated. With higher stable nuclei the energy of the photons required to disintegrate also increases. The low binding nuclei like ${ }_{1}^{2} \mathrm{H}$ and ${ }_{4}^{9} \mathrm{Be}$ are well-known to undergo photo-disintegration with $2.22 \mathrm{MeV}$ and $2.62 \mathrm{MeV}$ photons respectively [1]. Similarly, stimulated decay of neutrons as represented in Fig. 12 is also a well-known phenomenon. The prediction and its quantitative treatment can be done by hadronic mechanics.

According to Prof. Santilli, neutron is an unlimited source of energy because it decays releasing highly energetic electron and neutrino that can be easily trapped with a metal shield. It is known that an isolated neutron is highly unstable and has half life of approximately 15 minutes. However, as a constituent of nuclei, it shows high stability which has been attributed to a strong nuclear force of attraction. The neutron shows stimulated decay as

$$
\mathrm{TR}+\mathrm{n} \rightarrow \mathrm{p}^{+}+\beta^{-}
$$

where $\beta$ - has spin zero for the conservation law of the angular momentum. $\beta$ - can also be considered either as an electron and a neutrino or as an electron and an antietherino with opposing spin $1 / 2$. However, this difference is irrelevant for the stimulated decay of the neutron.

When a resonating photon hits a nucleus, it excites the isoelectron inside a neutron irrespective of whether the photon penetrates or not inside the neutron. The excited isoelectron leaves the neutron structure, thus causing its stimulated decay. This is due to the fact that hadronic mechanics predicts only one energy level for the proton and the electron in conditions of total mutual immersion (as incase of neutron). Range of hadronic mechanics is given by the radius of neutron that is 1 fm. Thus, the excited isoelectron excites the proton and reassumes its conventional quantum features when moving in vacuum.

Numerous additional triggers are predicted by hadronic mechanics such as photons with a wavelength equal to the neutron size. Here, the whole neutron is excited, rather than the isoelectron in its interior, but the result is always the stimulated decay.

\section{Double Beta Decay}

In this typical example of double decay first reaction is stimulated and the second is spontaneous [11].

$$
\begin{aligned}
& \gamma_{\mathrm{r}}(0,0,1)+\mathrm{N}(\mathrm{A}, \mathrm{Z}, \mathrm{J}) \rightarrow \\
& \mathrm{N}(\mathrm{A}, \mathrm{Z}+1, \mathrm{~J}+1)+\beta^{-}(0,-1,0) \\
& \mathrm{N}(\mathrm{A}, \mathrm{Z}+1, \mathrm{~J}+1) \rightarrow \\
& \mathrm{N}(\mathrm{A}, \mathrm{Z}+2, \mathrm{~J}+2)+\beta^{-}(0,-1,0)
\end{aligned}
$$

The original isotope should admit stimulated decay of at least one of its peripheral neutrons via one photon with a resonating frequency verifying all conservation laws of the energy, angular momentum, etc. The new nucleus formed should undergo spontaneous beta decay so that with one resonating photon there is production of two electrons whose kinetic energy is trapped with a metal shield to produce heat. The original isotope is metallic so that, following the emission of two electrons, it acquires an electric charge suitable for the production of a DC current between the metallic isotope and the metallic shield. The energy balance is positive. The initial and final isotopes are light, natural and stable elements so that the new energy is clean (since the electrons can be easily 
trapped with a thin metal shield), and produce non-radioactive waste.

E.g. double beta decay of the $\operatorname{Mo}(100,42,0)$

$$
\begin{aligned}
& \gamma_{\mathrm{r}}(0,0,1)+\operatorname{Mo}(100,42,0) \\
& \rightarrow \operatorname{Tc}(100,43,1)+\beta^{-}(0,-1,0) \\
& \operatorname{Tc}(100,43,1) \rightarrow \\
& \quad \operatorname{Ru}(100,44,0)+\beta^{-}(0,-1,0)
\end{aligned}
$$

$\operatorname{Mo}(100,42,0)$ is naturally stable with mass 99.9074771 amu. Tc $(100,43)$ has mass 99.9076576 amu and is naturally unstable with spontaneous decay into $\mathrm{Ru}(100,44,0)$ and half life of $15.8 \mathrm{~s}$. $\mathrm{Ru}(100,44)$ is naturally stable with mass $99.9042197 \mathrm{amu}$. Although the mass of $\operatorname{Mo}(100,42,0)$ is smaller than that of $\operatorname{Tc}(100,43,1)$, yet the conservation of energy can be verified with a resonating frequency of 0.16803 $\mathrm{MeV}$ (obtained for $\mathrm{n}=1 / 7$ ) where $\mathrm{n}$ is normalization contant.

But the mass of the original isotope is bigger than that of the final isotope for a value much bigger than that of the resonating photon, with usable hadronic energy (HE) power nuclear reaction

$$
\begin{aligned}
\mathrm{HE}= & \mathrm{M}(100,42)-\mathrm{M}(100,44)- \\
& \mathrm{E}(\gamma)-2 \times \mathrm{E}(\mathrm{e}) \\
= & 3.034-0.184-1.022 \mathrm{MeV} \\
= & 1.828 \mathrm{MeV}
\end{aligned}
$$

where Santilli subtracts the conventional rest energy of the two electrons because it is not usable as a source of energy in this case.

Under the assumptions of using a coherent beam with resonating photons hitting a sufficient mass of $\operatorname{Mo}(100,42,0)$ suitable to produce $10^{20}$ stimulated nuclear transmutations per hour, we have the following:

Hadronic production of heat :

$$
2 \times 10^{20} \mathrm{MeV} / \mathrm{h}=3 \times 10^{4} \mathrm{BTU} / \mathrm{h},
$$

Hadronic production of electricity :

$$
2 \times 10^{20} \mathrm{e} / \mathrm{h}=200 \mathrm{C} / \mathrm{h}=55 \mathrm{~mA} .
$$

\section{Applications of Hadronic Nuclear Energy}

\subsection{Intermediate Controlled Nuclear Synthesis}

1. Green power generation source

ICNS can be industrially exploited for power generation. Since there are no ionizing radiations or particular emissions, it is green and can be used for sustainable development.

2. Synthesis of heavy and super heavy elements

Synthesis of heavy elements particularly of the seventh period is conventionally done by bombarding two heavy nuclei. The reacting rather bombarding nuclei have high energy to overcome the coulombic barrier. This results in high excitation energy of the resulting daughter nuclei which is often higher than the fission barrier. Thus, fission is one of the pre-dominant exit channel.

However, if ICNS is used then the nuclei of the participating atoms can be exposed in controlled manner as well as can be brought near each other to a considerable extent without initiating coulombic repulsion. This is due to magnetic bond in the participating nuclei which in this case are magnecules rather than mere atoms or ions. The trigger mechanism then pushes the participating nuclei within hadronic radius where fusion is inevitable. Consequently formation of heavy nucleus takes place. Converting heavy nucleus into respective magnecules requires high magnetic field and would be a costly affair. However, the daughter heavy nuclei produced would be considerable stable making the synthesis green and viable.

Stable heavy daughter nuclei formed would allow study of its actual chemical characteristic instead of predicting on the basis of periodic table and spectroscopic studies.

\subsection{Particulate Type Nuclear Energy}

1. Green power generation source Stimulated decay of neutron and double beta decay can be used for power generation. The by product is electron which can be stopped with a metal sheet. This results in a clean and green power source.

2. Recycling of nuclear waste

It may also be used for recycling of nuclear waste generated due to existing conventional nuclear energy facility by stimulated neutron decay using photon with resonating frequency (or energy) of $1.294 \mathrm{MeV}$.

\section{Conclusion}

ICNS seems to be more promising than hot or cold fusion in terms of reproducibility and energy input to output ratio. The successful achievement of ICNS with industrial relevance depends on the proper selection of the hadronic fuel. The hadronic fuel is mainly due to-

a) The original and final nuclides are light, natural and stable isotope.

b) The nuclear syntheses cause no emission of ionizing radiations.

c) The energy produced $\Delta \mathrm{E}$ is much bigger than the total energy used by the equipment for its production.

Stimulated beta decay and double beta decay also seems to be promising prospect for green power generation. Apart from power generation, ICNS and stimulated decay holds promising prospect for synthesis of heavy elements and recycling of nuclear wastes respectively.

\section{Acknowledgement}

The financial support for this work from The R. M. Santilli Foundation is gratefully acknowledged. The author expresses her deep gratitude to Professor R. M. Santilli, Professor R. 
Anderson and Professor A. A. Bhalekar for encouragements and valuable guidance in preparing this paper.

\section{References}

[1] D. D. Sood, A. V. R. Reddy and N. Ramamoorthy; Fundamentals of Radiochemistry; Indian Association of Nuclear Chemists and Allied Scientists; $3^{\text {rd }}$ Edition; (2007).

[2] http://webapps1.cityofchicago.org/landmarksweb/web/landma rkdetails.htm?lanId=1383 downloaded on 25-05-2015.

[3] Frontiers Research Highlights 1946-1996. p. 11. published by the Office of Public Affairs, Argonne National Laboratory, Argonne, IL.

[4] Experimental Breeder Reactor-I, U.S. Department of Energy National Laboratory

[5] Rich Michal, Fifty years ago in December: Atomic reactor EBR-I produced first electricity, American Nuclear Society, Nuclear News. November 2001.

[6] H. A. Bethe; Energy Production in Stars; Nobel Lectures, Physics 1963-1970, Elsevier Publishing Company, Amsterdam, (1972).

[7] J. D. Lawson, Some Criteria for a Power producing thermonuclear reactor, Proceedings of the Physical Society. Section B Volume 70 Number 1, December 1955.

[8] Fleischmann, M., S. Pons, and M. Hawkins, Electrochemically induced nuclear fusion of deuterium. J. Electroanal. Chem., 1989. 261: p. 301.

[9] R. M. Santilli; Foundations of Hadronic Chemistry with applications to New Clean Energies and Fuels; Kluwer Academic Publishers, Netherlands, 2001
[10] A. K. Aringazin and M. G. Kucherenko; Exact variational solution of the restricted three-body Santilli-Shillady model of the hydrogen molecule; Hadronic Journal, 23, 1-56, (2000). (physics/0001056).

[11] I. Gandzha and J. Kadeisvily; New Sciences for a New Era: Mathematical, Physical and Chemical Discoveries of Ruggero Maria Santilli; San-Marino draft dated June 28, 2011.

[12] R. M. Santilli; The novel "Controlled Intermediate Nuclear Fusion" and a report on its industrial realization as predicted by Hadronic Mechanics; Hadronic Journal, 31, 115-200, (2008).

[13] M. G. Kucherenko and A. K. Aringazin; Estimate of the polarized magnetic moment of the isoelectronium in the hydrogen molecule; Hadronic Journal, 21, 895-902, (1998).

[14] A. K. Aringazin; Toroidal configuration of the orbit of the electron of the hydrogen atom under strong external magnetic fields; Hadronic Journal, 24, 395-434, (2001).

[15] R. M. Santilli; Experimental confirmation of Nitrogen synthesis from deuterium and carbon without harmful radiations; New Advances in Physics, 5, 29-36, (2011).

[16] R. M. Santilli; Additional Confirmation of the "Intermediate Controlled Nuclear Fusions" without harmful radiation or waste; Proceedings of the Third International Conference on the Lie-Admissible Treatment of Irreversible Processes, Kathmandu University; Nepal, pp. 163-177, (2011).

[17] J. V. Kadeisvili, C. Lynch and Y. Yang; Confirmations of Santilli's Intermediate Controlled Nuclear Fusion of Deuterium and Carbon into Nitrogen without harmful radiations; The Open Physical Chemistry Journal, 5, 17-27, (2013). 